\title{
Recurrent Chronic Obstructive Pulmonary Disease Exacerbations after Endobronchial Valve Implantation Are Associated with the Presence of Pseudomonas aeruginosa
}

\author{
Izabela Tuleta $^{a}$ Carmen Pizarro $^{a}$ Ernst Molitor ${ }^{b}$ Glen Kristiansen ${ }^{c}$ \\ Georg Nickenig a Dirk Skowasch ${ }^{\mathrm{a}}$ \\ ${ }^{a}$ Department of Internal Medicine II - Cardiology and Pulmonology, ${ }^{b}$ Institute of Medical Microbiology, \\ Immunology and Parasitology, and CInstitute of Pathology, University of Bonn, Bonn, Germany
}

\section{Key Words}

Endoscopic lung volume reduction - Chronic obstructive pulmonary disease $\cdot$ Pseudomonas aeruginosa

\begin{abstract}
Background: Endoscopic lung volume reduction by means of endobronchial valve implantation is an established therapy in patients with severe emphysema. However, longterm complications such as chronic obstructive pulmonary disease (COPD) exacerbations are a limitation of this method. Objectives: As the mechanisms underlying increased rates of COPD exacerbations are unknown, the aim of our study was to determine whether infectious or inflammatory factors may contribute to these events and to investingate the consequent need for valve explantation. Methods: Tissue surrounding explanted endobronchial Zephyr valves was examined by microbiological, histological and cytological methods. Additionally, we performed a microbiological analysis of tracheal aspirates before both valve implantation and valve explantation. Moreover, blood samples were collected for the analysis of inflammatory markers. Results: Endobronchial valves were explanted from 16 patients. Reasons for explantation were frequent postprocedural COPD exacerbations (group 1: 8 patients) or loss of clinical benefit
\end{abstract}

(group 2: 8 patients). Compared to group 2, the microbiological examinations of valve lavage and tracheal aspirates from patients in group 1 showed a higher detection of Gramnegative bacteria. In particular, infection with Pseudomonas aeruginosa was more predominant in group 1 , while no presence could be detected in group 2. Blood inflammatory markers tended to be slightly higher in group 1 than in group 2; however, without reaching statistical significance. Conclusions: Increased rates of COPD exacerbations after endobronchial valve implantation are associated with the presence of $P$. aeruginosa. The finding warrants further investigation.

(c) 2016 S. Karger AG, Basel

\section{Introduction}

Endoscopic lung volume reduction (ELVR) by means of endobronchial valve implantation is an established therapy concept for selected patients with chronic obstructive pulmonary disease (COPD) and severe emphysema [1-5]. Endobronchial valve implantation results in improvement of clinical, lung function and hemodynamic parameters [6-10]. However, this procedure is not free of short- and long-term complications such as pneumothorax, hemop-

\section{KARGER}

E-Mail karger@karger.com

www.karger.com/res (c) 2016 S. Karger AG, Basel

0025-7931/16/0916-0510\$39.50/0 
Table 1. Baseline patient characteristics

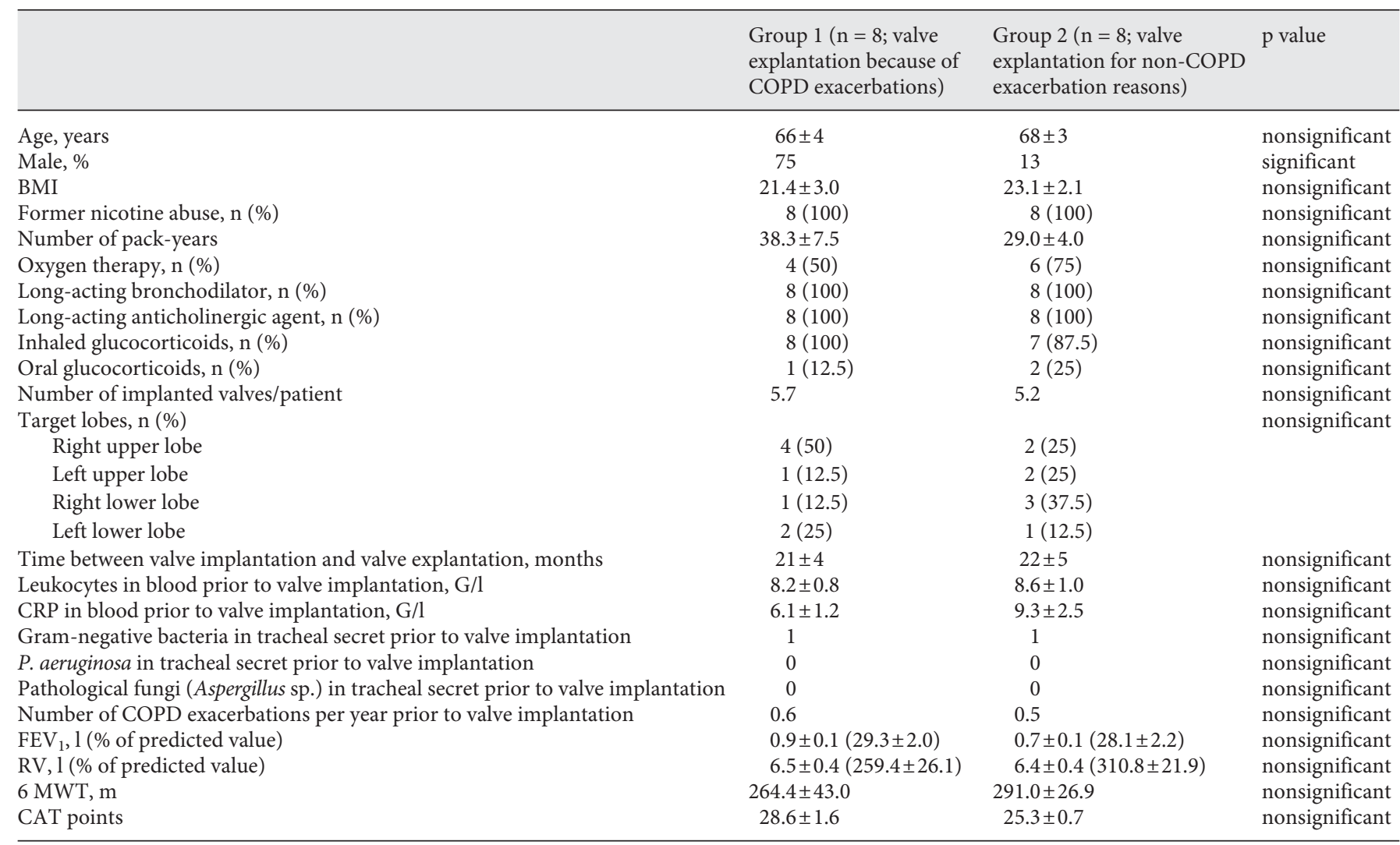

No significant differences in patient characteristics at baseline between the group of patients with valve explantation because of COPD exacerbations

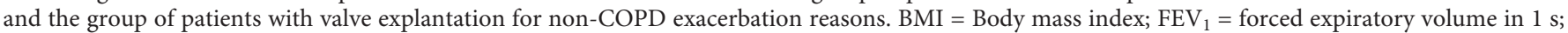
$\mathrm{RV}=$ residual volume; $6 \mathrm{MWT}=$ six-minute walk test; $\mathrm{CAT}=\mathrm{COPD}$ assessment test. $\mathrm{p}>0.05=$ Nonsignificant.

tysis, valve displacement, COPD exacerbations, pneumonia and death $[1,2,11-13]$. Patients who suffer from frequent COPD exacerbations prior to treatment should not be assigned to this procedure [14]. However, despite the selection of optimal candidates for valve implantation, COPD exacerbations and pneumonia are the most common valve-associated posttreatment side effects $[1,11]$. It is not understood why some patients develop these complications and others do not. Moreover, it is not known which factors, whether viral, bacterial, mycotic or noninfectious, contribute to the increased exacerbation rates.

Compared to other ELVR approaches, one advantage of endobronchial valve implantation therapy is that this form of treatment is reversible: for example, when patients experience too many COPD exacerbation episodes, do no longer benefit from the therapy or for any other medical reason, the valves can be easily explanted during a simple bronchoscopy procedure.

Detection of $P$. aeruginosa after

Endobronchial Valve Implantation
Therefore, the aims of our present study were the examinations of microbiological, histological and cytological characteristics of the tissue surrounding explanted valves, a comparison of the microbiological endobronchial status and the levels of blood inflammatory markers over the time course between valve implantation and valve explantation, as well as the assessment of possible correlations between these findings and the underlying reasons for valve removal.

\section{Material and Methods}

\section{Study Design}

In our hospital, we have explanted endobronchial valves from 16 patients with emphysema included in this study between May 2014 and December 2015, whereas we had conducted ELVR with endobronchial valves in 87 patients since October 2011. Sixteen patients had their endobronchial valves removed, i.e. 8 patients for too fre- 
Table 2. Follow-up patient characteristics before valve explantation

\begin{tabular}{lcc}
\hline & $\begin{array}{l}\text { Group 1 }(\mathrm{n}=8 \text {; valve } \\
\text { explantation because of } \\
\text { COPD exacerbations) }\end{array}$ & $\begin{array}{l}\text { Group 2 (n = 8; valve } \\
\text { explantation for non-COPD } \\
\text { exacerbation reasons) }\end{array}$ \\
\hline FEV $_{1}, 1$ (\% of predicted value) & $0.8 \pm 0.0(24.1 \pm 1.5)$ & $0.6 \pm 0.0(28.0 \pm 3.2)$ \\
RV, 1 (\% of predicted value) & $6.4 \pm 0.3(249.1 \pm 18.3)$ & $6.3 \pm 1.2(294.2 \pm 30.0)$ \\
6 MWT, m & $300.0 \pm 70.4$ & $321.3 \pm 40.9$ \\
CAT points & $30.5 \pm 1.4$ & $28.3 \pm 1.5$ \\
\hline
\end{tabular}

Compared to baseline characteristics, there are no lung function or clinical improvements in either of the two groups of patients. Of note, lung function and clinical parameters were measured in patients from group 2 in a COPD exacerbation-free interval. $\mathrm{FEV}_{1}=$ Forced expiratory volume in $1 \mathrm{~s}$; RV = residual volume; $6 \mathrm{MWT}=$ sixminute walk test; CAT $=$ COPD assessment test.

quent COPD exacerbation episodes (for a definition see below, group 1) and 8 for loss or insufficient clinical benefits (group 2). Except for sex, with a predominance of men in group 1, there were no significant differences between the groups at baseline, as presented in table 1. Microbiological, histological and cytological analyses of tracheal aspirates and lavage of explanted valves were conducted. Additionally, inflammatory markers were examined in blood.

\section{Patient Characteristics and Bronchoscopic Procedures}

The research was conducted according to the principles of the Declaration of Helsinki and approved by the local ethics committee. Patients with COPD and severe heterogeneous emphysema under optimal drug therapy underwent ELVR by means of endobronchial valve implantation (Zephyr EBV; Pulmonx Inc., Redwood City, Calif., USA) in our hospital. The presence of collateral ventilation in the target lobe was excluded by the Chartis bronchoscopy physiological mapping system (Pulmonx Inc.). The clinical files of all patients who had their valves explanted were collected and studied.

Sixteen patients had their valves removed. In this cohort, endobronchial valves were placed into the right upper lobe in 6 patients, the left upper lobe in 3 patients, the right lower lobe in 4 patients and the left lower lobe in 3 patients. In 7/16 patients, in whom the valves could not be explanted within one session, we performed additional bronchoscopic procedures until all valves were removed.

Following intervention, all patients were examined every 3 months on average for clinical, functional and morphological lung changes and inflammatory blood markers.

Since it is known that the COPD exacerbation rates are higher 3 months after valve implantation compared to the further time course [1], we have accepted up to 1 COPD exacerbation per month in the first 3 months following the procedure and treated these patients with antibiotics and cortisone (group 1: 5 patients; group 2: 2 patients). The only exception of this concept was 1 patient in group 1 who experienced a persistent COPD exacerbation state despite adequate medical treatment and underwent valve explantation 2 months after ELVR. After a period of 3 months, patients from group 1 had to suffer from at least 3 clinically significant COPD exacerbations in the consecutive 6 months in spite of optimal drug treatment to be assigned to the valve explantation

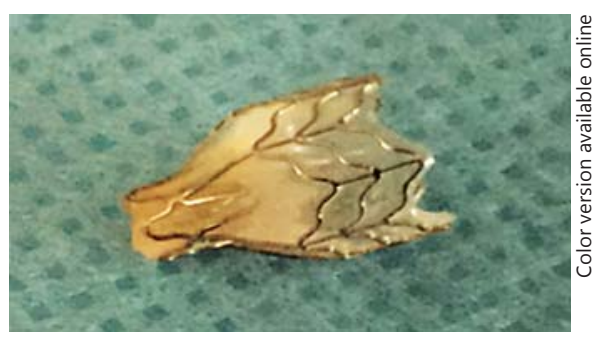

Fig. 1. An exemplary photo of the explanted valve with a newly formed tissue between the struts.

procedure. The patients from group 2 did not have any relevant COPD exacerbations (COPD exacerbation rate $<1$ per 6 months).

Of note, ELVR did not improve the clinical status or lung function parameters in either of the two groups prior to valve explantation (table 2).

\section{Valve Explantation with Subsequent Microbiological,}

Histological and Cytological Analyses

The explantation of the valves occurred without relevant complications, such as significant bleeding, perforation, pneumothorax or death. The explanted valves were rinsed immediately in physiological sodium chloride solution and gently shaken to collect the tissue attached to the valve struts in the surrounding solution. The specimens were analyzed in our microbiological, histopathological and biochemistry laboratories according to standard procedures. The aim of these examinations was to assess and quantify the pathogen burden and potential inflammatory reactions. In addition, microbiological tests of tracheal aspirates were performed before both valve implantation and valve explantation.

\section{Blood Tests}

Blood samples were collected before both valve implantation and valve explantation for the analysis of inflammatory response [leukocyte number and C-reactive protein (CRP) levels] according to standard laboratory tests. 


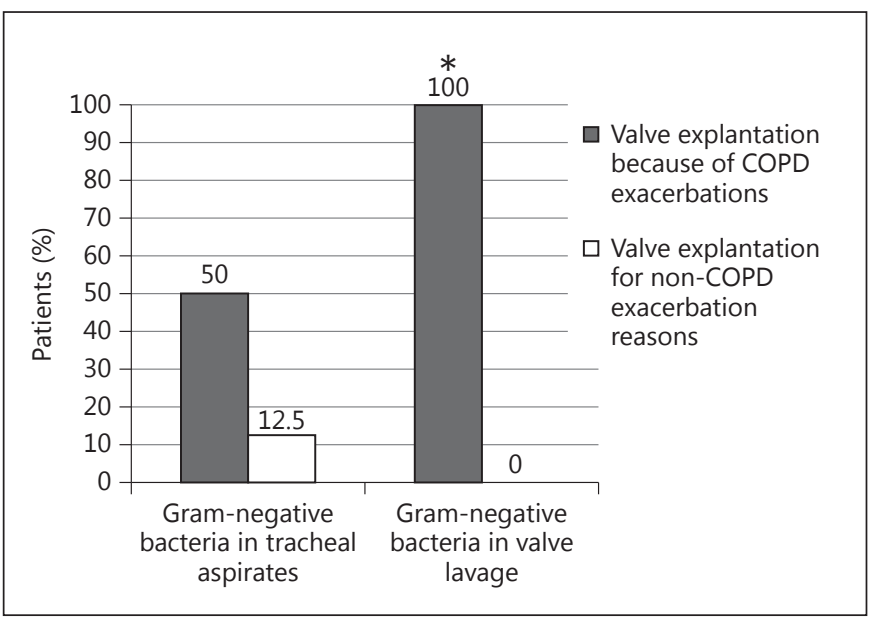

Fig. 2. A higher number of patients with COPD-related valve explantations present Gram-negative bacteria in tracheal aspirates and valve lavage than patients with valve explantation for nonCOPD exacerbation reasons. ${ }^{*} \mathrm{p}<0.05$.

\section{Statistical Analysis}

Potential differences in parameter means between both groups were assessed by Student's t test. Categorical variables were compared by means of the $\chi^{2}$ test. $p$ values $<0.05$ were considered statistically significant.

\section{Results}

Valves were explanted after a mean time of approximately 22 months in both groups (range $2-45$ months). The explanted endobronchial valves carrying newly formed tissue (fig. 1) were subsequently analyzed by microbiological, cytological and histological methods. All patients in group 1 presented an increased level of Gramnegative bacteria (fig. 2) compared to group 2. There was also a clear predominance of Pseudomonas aeruginosa (fig. 3 ) in valve lavage and tracheal aspirates before valve explantation in group 1, while there were barely relevant pathogens and, in particular, no detectable presence of $P$. aeruginosa in group 2. Interestingly, in the patients of group 1, the microbe burden of $P$. aeruginosa was higher in valve lavage than in the tracheal secret. Moreover, a greater variety of pathogens could be identified in valve lavage versus tracheal aspirates ( $P$. aeruginosa, Serratia marcescens, Serratia rubidacea, Klebsiella oxytoca, Acinetobacter baumannii vs. P. aeruginosa, S. marcescens, Klebsiella pneumoniae, respectively).

Detection of $P$. aeruginosa after Endobronchial Valve Implantation

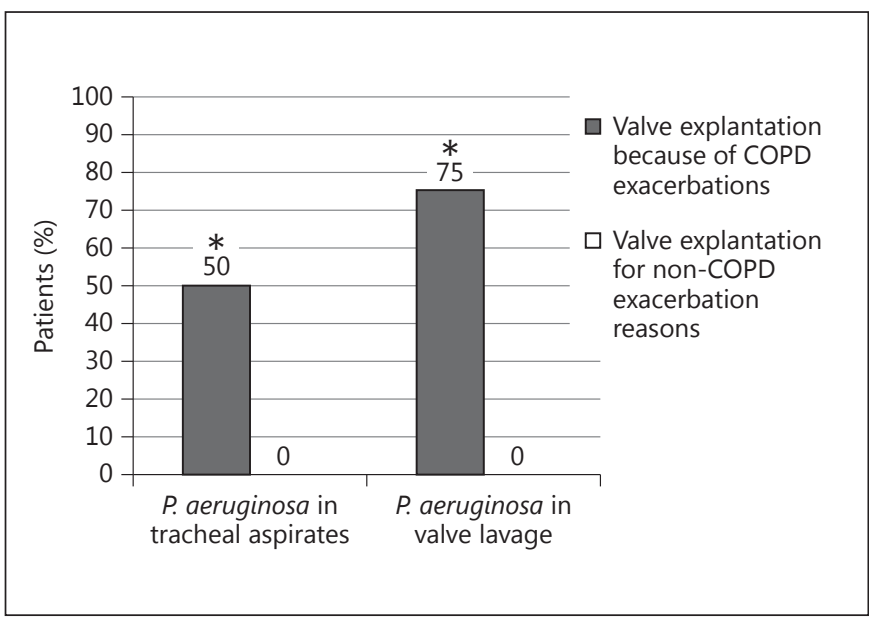

Fig. 3. Predominant presence of $P$. aeruginosa in tracheal aspirates and valve lavage in patients with valve explantation because of COPD exacerbations. No detection of $P$. aeruginosa in tracheal aspirates and valve lavage in patients with valve explantation for reasons other than COPD exacerbation. ${ }^{*} \mathrm{p}<0.05$.

In accordance with the significantly greater pathogen burden in group 1 than in group 2, a semiquantitative histological method demonstrated a moderate to pronounced inflammation in all specimens from group 1 ( $\mathrm{n}=8,100 \%$; fig. 4). In contrast, the specimens from group 2 either lacked inflammation or showed only a slight inflammatory reaction $(\mathrm{n}=7,87.5 \%)$.

Furthermore, microbiological examination of the preimplantation tracheal aspirates showed no relevant pathogen growth, especially no $P$. aeruginosa detection in any of the patients who underwent a subsequent valve explantation. Of note, there was no correlation between the number of granulocytes and lymphocytes from valve lavage or the leukocytes/CRP levels in serum measured before valve explantation and the presence of COPD exacerbations/endobronchial pathogen burden. The mean concentration of granulocytes and lymphocytes from valve lavage in the cytological analysis was $518 \pm 237 / \mu \mathrm{l}$ and $86 \pm 47 / \mu \mathrm{l}$, respectively, in group 1 , and $827 \pm 502 / \mu \mathrm{l}$ and $143 \pm 69 / \mu \mathrm{l}$, respectively, in group 2, without statistical significance between the groups. The average CRP levels at the time of valve explantation were greater in group 1 than in group 2 (22.9 \pm 9.8 vs. $8.9 \pm 2.7 \mathrm{mg} / \mathrm{l})$, but this difference was not statistically significant. The number of blood leukocytes before valve explantation was similar in both groups $(9.7 \pm 1.2 \mathrm{vs}$. $8.0 \pm 0.6 \mathrm{G} / \mathrm{l}$, respectively; fig. 5). The average number of implanted valves per patient did not correlate with the valve-associated infectious complications. 

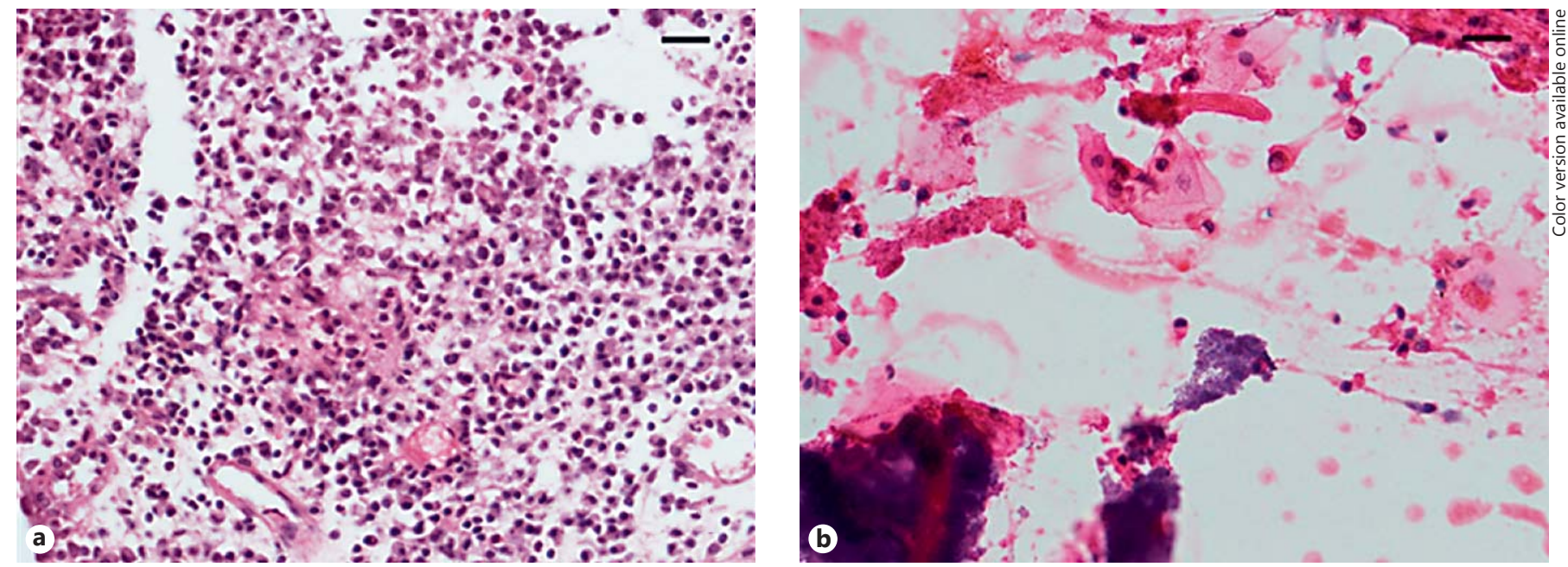

Fig. 4. Representative photomicrographs of the hematoxylin and eosin-stained tissue surrounding explanted valves obtained by light microscopy. a Tissue with moderate to pronounced inflammatory reaction adherent to the explanted valve from a patient with valve explantation because of COPD exacerbations. $\mathbf{b}$ No relevant inflammation around the explanted valve in a patient without COPD exacerbations after valve implantation. Bar $=30 \mu \mathrm{m}$.

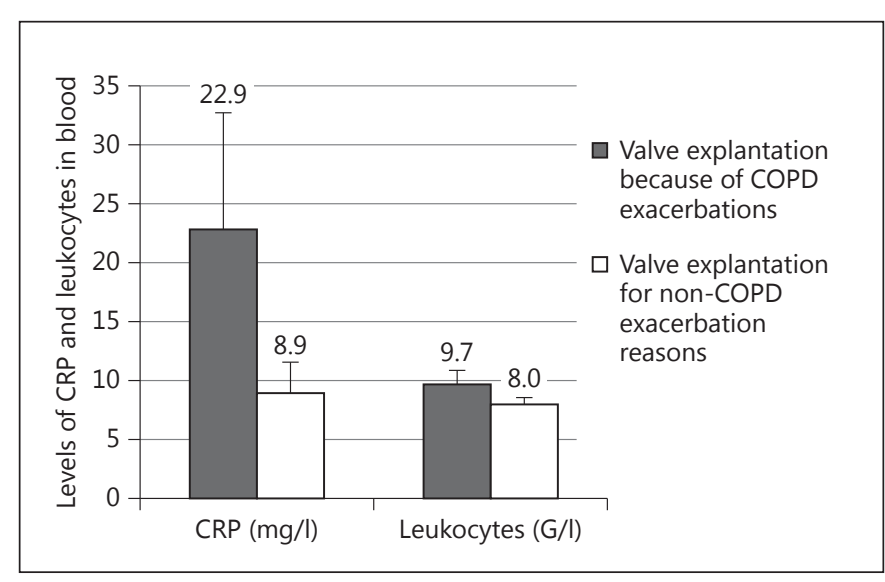

Fig. 5. Tendency towards higher CRP concentrations in blood in patients with valve explantation because of COPD exacerbations than in patients with valve explantation for reasons other than COPD exacerbation. No relevant differences in the levels of leukocytes between the two groups.

\section{Discussion}

Based on the existing studies, ELVR by means of endobronchial valve implantation may be an effective and safe therapy in patients with severe emphysema characterized by an absence of interlobar collateral ventilation
$[4,5,9]$. However, some adverse events may limit the efficacy of this treatment option $[4,5,9]$. The main finding of our present study of patients with severe emphysema was the de novo presence of endobronchial Gram-negative bacteria after valve implantation that was associated with frequent COPD exacerbations requiring valve explantations. The predominant pathogen detected was $P$. aeruginosa. In contrast, the tracheobronchial fluids obtained from patients in whom valve explantation was indicated for reasons other than COPD exacerbation were free of significant pathogens, especially of $P$. aeruginosa. The analysis of the tracheal secret before valve implantation in all patients studied showed no growth of clearly pathogenic microbes. This finding suggests that a new infection with Gram-negative bacteria, especially P. aeruginosa, following valve implantation predicts postprocedural COPD exacerbations and the resultant need for valve explantation.

Similar observations have been reported by other groups for COPD patients without ELVR. Herein, the isolation of $P$. aeruginosa from sputum samples of COPD patients suggested that this bacterium could be a common cause of infection $[15,16]$ that may lead to COPD exacerbations in this patient population [17]. Interestingly, increased airway bacterial colonization rates, especially with Gram-negative bacteria, were detected in patients who underwent lung transplantation after ELVR com- 
pared to lung transplant patients without prior ELVR [18]. Since the baseline characteristics of the enrolled patients from both groups in our study showed no relevant differences, except for sex, it is difficult to determine which potential risk factors are connected to the later COPD exacerbations underlying the failure of the endobronchial interventional therapy. It is known that frequent COPD exacerbations contraindicate ELVR [2]. However, in our study, the selected patients for the subsequent valve implantation were not characterized by COPD exacerbations in the past and were free of endobronchial pathogens or clinically relevant increased inflammatory markers in blood at the time of the implantation procedure.

Our next finding is that the qualitative and the semiquantitative microbiological analysis of tracheal aspirates and lavage from the explanted valves showed a significantly greater number and variety of pathogen species in valve lavage compared to the tracheal aspirates. This suggests that control bronchoscopy with the collection of tracheal secret for microbiological testing, in the context of either COPD exacerbation- or other reason-related valve explantation, may not always adequately mirror the degree of the real infection local to the valve. Therefore, a deeper sample collection, for example from the valve bronchus during control bronchoscopy, is possibly of greater value for the detection of pathogens. Following the microbiological results and the clinical response to ELVR, valve explantation or antibiotic treatment could be evaluated in an individualized discussion. The histological detection of significantly aggravated inflammation in explanted valve specimens from patients with COPD exacerbations as a reason for valve explantation suggests that an endobronchial superficial biopsy of the bronchial mucosa close to the valve sites could be indicated, in addition to microbiological examinations, to follow up patients at risk of COPD exacerbations after endobronchial valve therapy. In contrast, the sole interpretation of the cytological findings regarding the number of inflammatory cells in valve lavage does not distinguish between patients with or without COPD exacerbations after valve treatment, given that differences were not observed in the levels of granulocytes/lymphocytes in valve lavage between these two patient cohorts. Similarly, the lack of significant differences in the CRP and leukocyte levels in serum shortly before valve explantation between the two patient groups makes these standard blood inflammatory markers unsuitable for predicting COPD exacerbation following an endobronchial valve procedure.

Despite the fact that our present study is limited to a small number of enrolled patients and a true cause-effect relationship regarding bacteria detection and increased COPD exacerbation rates cannot be validated by our findings, it provides new data that may be helpful in the early identification of patients at risk of COPD exacerbations after ELVR. Avoiding clinically relevant COPD exacerbations could prevent further deterioration of the lung in patients with already strongly reduced respiratory reserve. Therefore, further multicenter studies are mandatory to better understand the issues of ELVR therapy in order to maximize the benefits and to minimize the potential complications of this treatment.

\section{Acknowledgements}

We would like to thank Sabine Ring for her excellent technical assistance.

\section{References}

$>1$ Sciurba FC, Ernst A, Herth FJ, Strange C, Criner GJ, Marquette CH, Kovitz KL, Chiacchierini RP, Goldin J, McLennan G; VENT Study Research Group: A randomized study of endobronchial valves for advanced emphysema. N Engl J Med 2010;363:1233-1244.

-2 Davey C, Zoumot Z, Jordan S, McNulty WH, Carr DH, Hind MD, Hansell DM, Rubens MB, Banya W, Polkey MI, Shah PL, Hopkinson NS: Bronchoscopic lung volume reduction with endobronchial valves for patients with heterogeneous emphysema and intact interlobar fissures (the BeLieVeR-HIFi study): a randomised controlled trial. Lancet 2015;386:1066-1073
3 Pizarro C, Ahmadzadehfar H, Essler M, Tuleta I, Fimmers R, Nickenig G, Skowasch D: Effect of endobronchial valve therapy on pulmonary perfusion and ventilation distribution. PLoS One 2015;10:e0118976.

$\checkmark 4$ Klooster $\mathrm{K}$, ten Hacken NH, Hartman JE, Kerstjens HA, van Rikxoort EM, Slebos DJ: Endobronchial valves for emphysema without interlobar collateral ventilation. N Engl J Med 2015;373:2325-2335.

5 Park TS, Hong Y, Lee JS, Oh SY, Lee SM, Kim N, Seo JB, Oh YM, Lee SD, Lee SW: Bronchoscopic lung volume reduction by endobronchial valve in advanced emphysema: the first Asian report. Int J Chron Obstruct Pulmon Dis 2015;10:1501-1511.
6 Pietzsch JB, Garner A, Herth FJ: Cost-effectiveness of endobronchial valve therapy for severe emphysema: a model-based projection based on the VENT study. Respiration 2014; 88:389-398.

$>7$ Koegelenberg CF, Slebos DJ, Shah PL, Theron J, Dheda K, Allwood BW, Herth FJ: Time for the global rollout of endoscopic lung volume reduction. Respiration 2015;90:430-440.

$>8$ Eberhardt R, Herth FJ, Radhakrishnan S, Gompelmann D: Comparing clinical outcomes in upper versus lower lobe endobronchial valve treatment in severe emphysema. Respiration 2015;90:314-320. 
9 Choi M, Lee WS, Lee M, Jeon K, Sheen S, Jheon S, Kim YS: Effectiveness of bronchoscopic lung volume reduction using unilateral endobronchial valve: a systematic review and meta-analysis. Int J Chron Obstruct Pulmon Dis 2015;10:703-710.

10 Eberhardt R, Gerovasili V, Kontogianni K, Gompelmann D, Ehlken N, Herth FJ, Grünig E, Nagel C: Endoscopic lung volume reduction with endobronchial valves in patients with severe emphysema and established pulmonary hypertension. Respiration 2015;89: 41-48.

11 Gompelmann D, Eberhardt R, Herth F: Endoscopic volume reduction in COPD - a critical review. Dtsch Arztebl Int 2014;111:827-833.
12 Gompelmann D, Herth FJ, Slebos DJ, Valipour A, Ernst A, Criner GJ, Eberhardt R: Pneumothorax following endobronchial valve therapy and its impact on clinical outcomes in severe emphysema. Respiration 2014;87:485-491.

13 Valipour A, Slebos DJ, de Oliveira HG, Eberhardt R, Freitag L, Criner GJ, Herth FJ: Expert statement: pneumothorax associated with endoscopic valve therapy for emphysema - potential mechanisms, treatment algorithm, and case examples. Respiration 2014;87:513-521.

14 de Macedo-Neto AV, de Oliveira HG: Transbronchoscopic emphysema treatment with one-way Zephyr valves; in Díaz-Jimenez JP, Rodriguez AN (eds): Interventions in Pulmonary Medicine. Springer, New York, 2013, chapt 27, p 381.
15 Groenewegen KH, Wouters EF: Bacterial infections in patients requiring admission for an acute exacerbation of COPD; a 1-year prospective study. Respir Med 2003;97:770-777.

16 Martínez-Solano L, Macia MD, Fajardo A, Oliver A, Martinez JL: Chronic Pseudomonas aeruginosa infection in chronic obstructive pulmonary disease. Clin Infect Dis 2008;47: 1526-1533.

17 Murphy TF, Brauer AL, Eschberger K, Lobbins P, Grove L, Cai X, Sethi S: Pseudomonas aeruginosa in chronic obstructive pulmonary disease. Am J Respir Crit Care Med 2008;177: 853-860.

18 Fuehner T, Clajus C, Fuge J, Jonigk D, Welte T, Haverich A, Greer M, Gottlieb J: Lung transplantation after endoscopic lung volume reduction. Respiration 2015;90:243-250. 